\title{
Single versus mixed edge activators in caffeine-loaded transfersomes: physicochemical and cytotoxicity assessment
}

\author{
Ativadores de superficie únicos versus mistos em transferossomas para veicular cafeína: avaliação \\ fisico-química e de citotoxicidade
}

\begin{abstract}
Íris Guerreiro ${ }^{1,2 \#}$, Marta Rodrigues ${ }^{3 \#}$, Ana Sofia Fernandes ${ }^{1}$, Catarina Rosado ${ }^{1}$, Catarina Pereira-Leite ${ }^{1,4}$ *
${ }^{1}$ CBIOS - Universidade Lusófona's Research Center for Biosciences \& Health Technologies, Av Campo Grande 376, 1749-024 Lisboa, Portugal; ${ }^{2}$ Department of Biomedical Sciences, University of Alcalá, Ctra. Madrid-Barcelona Km. 33.600, 28871 Alcalá de Henares, Madrid, Spain; ${ }^{3}$ Escola de Psicologia e Ciências da Vida, Universidade Lusófona de Humanidades e Tecnologias, Av Campo Grande 376, 1749-024 Lisboa, Portugal; ${ }^{4}$ LAQV, REQUIMTE, Departamento de Ciências Químicas, Faculdade de Farmácia, Universidade do Porto, Rua de Jorge Viterbo Ferreira 228, 4050-313 Porto, Portugal
\end{abstract}

\# These authors contributed equally to this work.

*corresponding author / autor para correspondência: catarina.leite@ulusofona.pt

\begin{abstract}
The performance of transfersomes as nanovesicular systems to enhance the skin permeation of bioactive compounds may be modulated according to their composition of edge activators(EAs). Vesiclesize, deformability, and encapsulation efficiency can be altered when using nonionic surfactants as EAs with opposing hydrophilic-lipophilic balance (HLB). Thus, this work aimed to assess the impact on the physicochemical properties and the in vitro cytotoxicity profile of transfersomal formulations composed of single or mixed EAs, with opposing HLB values. Transfersomes made of single or mixed nonionic surfactants (Tween ${ }^{\mathbb{B}} 80$ and/or $\operatorname{Span}^{\circledR} 80$ ) were prepared in the presence and absence of caffeine, used as a model hydrophilic compound. Single or mixed EAs-containing transfersomes displayed promising physicochemical properties for cutaneous applications and were found to be stable for one month of refrigerated storage. Moreover, all transfersomal formulations were compatible with a keratinocyte cell line upon $24 \mathrm{~h}$ treatment. Altogether, these preliminary data suggest that the use of mixed nonionic surfactants as EAs may be further explored to modulate the performance of transfersomes as skin permeation enhancers.
\end{abstract}

Keywords: Vesicular systems, nanotechnology, nonionic surfactants, caffeine

\begin{abstract}
Resumo
O desempenho dos transferossomas como sistemas nanovesiculares para melhorar a permeação cutânea de compostos bioativos pode ser modulado de acordo com a sua composição em ativadores de superfície (EAs). Segundo a literatura, o tamanho, a deformabilidade e a eficiência de encapsulação destas vesículas dependem do equilíbrio hidrofílicolipofilico (HLB) dos tensioativos não-iónicos usados como EAs. Assim, este trabalho teve como objetivo avaliar o impacto nas propriedades físico-químicas e no perfil de citotoxicidade in vitro de formulações transferossomais constituídas por EAs únicos ou mistos, com valores de HLB opostos. Os transferossomas obtidos com tensioativos não-iónicos únicos ou mistos (Tween ${ }^{\circledR} 80$ e/ou Span ${ }^{\circledR} 80$ ) foram preparados na presença e ausência de cafeína, utilizada como composto modelo hidrofílico. Os transferossomas preparados, independentemente da sua composição em EAs, apresentaram propriedades físico-químicas promissoras para aplicações cutâneas, sendo estáveis durante 1 mês sob armazenamento refrigerado. Além disso, todas as formulações transferossomais foram compatíveis com uma linha celular de queratinócitos após tratamento por 24 h. No conjunto, estes dados preliminares sugerem que a utilização de tensioativos não-iónicos mistos como EAs poderá ser mais explorada para modular o desempenho dos transferossomas como promotores da permeação cutânea.
\end{abstract}

Palavras-chave: Sistemas vesiculares; nanotecnologia; tensioativos não-iónicos; cafeína 


\section{Introduction}

Skin is a highly efficient barrier against the external environment, controlling the penetration of xenobiotics and microorganisms to maintain homeostasis. The outermost layer of the epidermis is the stratum corneum (SC), consisting of a matrix of layers of flattened corneocytes, extracellularly surrounded by multiple lipid bilayers $(1,2)$. The unique structure of SC supports its primary function as cutaneous barrier, such that only a limited range of substances can permeate through skin, including substances with low molecular weight $(<500 \mathrm{Da})$, relative lipophilicity (log P $1-3)$, and water solubility (3). Thus, the number of therapeutic agents amenable to transdermal administration is limited, triggering the development of different vesicular systems, such as liposomes, ethosomes, niosomes, and transfersomes (3-6), to enhance the permeation of bioactive compounds into the skin.

Transfersomes are highly elastic and deformable vesicles, and as such, they have been viewed as the most advantageous vesicular systems to enhance skin permeation $(4,5)$. They were introduced by Cevc and Blume in 1992 (7) and are composed of phospholipids intercalated with edge activators (EAs) $(4,5)$. The incorporation of EAs in the formulation of transfersomes provides their most important characteristic - ultradeformability - as EAs act as destabilizers of the phospholipid bilayer by reducing interfacial tension (8). The ultra-elasticity of transfersomes allows them to deform and permeate through skin pores 5 to 10 times smaller than their size without losing their structure (3), and thus able to transport bioactive compounds to deeper skin layers. Transfersomes can be loaded with hydro- or lipophilic molecules with a wide range of molecular weights $\left(200-10^{6} \mathrm{Da}\right)$. Thus, they have been explored for encapsulation of diverse therapeutic agents, including antioxidant, anticancer, anti-inflammatory, analgesic, anesthetic, antidiabetic, and antimicrobial compounds (5).

The performance of transfersomes as permeation enhancers of bioactive compounds depends on the phospholipid:EA ratio and the chosen EA $(4,5)$.Various EAs have been explored to prepare transfersomes, in particular, nonionic surfactants $\left(\right.$ Tween $^{\circledR} 80$ and $\operatorname{Span}^{\circledR}$ 80 ) and bile acids (sodium deoxycholate and sodium cholate) (9-12). The choice of phospholipid:EA ratio is crucial to control the vesicle size and drug loading capacities, as recently reviewed $(4,5)$. In fact, this parameter should be optimized, preferably by qualityby-design strategies, as excessive amounts of EA (>

\section{Introdução}

A pele é uma barreira altamente eficaz contra o ambiente externo, controlando a penetração de xenobióticos e de microrganismos para manter a homeostase. A camada mais externa da epiderme é o estrato córneo (SC), constituído por uma matriz de camadas de corneócitos achatados, extracelularmente rodeados por múltiplas bicamadas lipídicas $(1,2)$. A estrutura única do SC está na base da sua função primária - funcionar como barreira cutânea, razão pela qual apenas uma gama limitada de substâncias pode permear através da pele, tais como substâncias com baixo peso molecular $(<500$ Da), lipofilia relativa ( $\log$ P 1-3) e solubilidade em água (3). Assim, o número de agentes terapêuticos que são passíveis de administração transdérmica é limitado, o que tem desencadeado o desenvolvimento de vários sistemas vesiculares para melhorar a permeação cutânea de compostos bioativos, tais como lipossomas, etossomas, niossomas e transferossomas (3-6).

Os transferossomas são vesículas altamente elásticas e deformáveis, razão pela qual parecem ser os sistemas vesiculares mais vantajosos para promover a permeação cutânea $(4,5)$. Foram introduzidos por Cevc e Blume (7) e são compostos por fosfolípidos intercalados com ativadores de superfície (EAs) $(4,5)$. A incorporação de EAs na formulação está na origem da sua característica mais importante - a ultra-deformabilidade, uma vez que os EAs atuam como destabilizadores da camada fosfolipídica ao reduzir a tensão interfacial (8). A ultra-elasticidade dos transferossomas permite-lhes deformar e permear através de poros cutâneos 5 a 10 vezes menores do que o seu tamanho sem perder a sua estrutura (3), sendo capazes de transportar compostos bioativos para as camadas cutâneas mais profundas. Os transferossomas podem veicular moléculas hidro- ou lipofílicas com uma vasta gama de pesos moleculares (200-10 $\left.{ }^{6} \mathrm{Da}\right)$. Por isso, têm vindo a ser explorados para encapsular diversos agentes terapêuticos, tais como compostos antioxidantes, anticancerígenos, antiinflamatórios, analgésicos, anestésicos, antidiabéticos e antimicrobianos (5).

O desempenho dos transferossomas como promotores de permeação de compostos bioativos depende da relação fosfolípido:EA e do EA escolhido $(4,5)$. Vários EAs têm sido explorados para preparar transferossomas, em particular, tensioativos não-iónicos $\left(\right.$ Tween $^{\circledR} 80$ e Span ${ }^{\circledR}$ 80) e ácidos biliares (desoxicolato de sódio e colato de sódio) (9-12). A escolha da relação fosfolípido:EA é crucial para controlar o tamanho da vesícula e a sua capacidade de carga, tal como foi recentemente revisto 
15\%) may lead to vesicle-to-micelle transitions (11). Moreover, the selection of EA in the preparation of transfersomes is a key factor to modulate their vesicle size and encapsulation efficiency. Considering nonionic surfactants, hydrophilic compounds may be more efficiently encapsulated using surfactants with high hydrophilic-lipophilic balance (HLB), while surfactants with low HLB values seem to better encapsulate lipophilic molecules (5). Moreover, the lower the HLB of nonionic surfactants, the higher the vesicle size (9) and the lower the vesicle deformability (10), which may ultimately reduce skin permeation. Altogether, these data indicate that modulating the HLB values by using mixtures of EAs may be a valuable strategy to optimize the performance of transfersomes.

In this context, this preliminary study aimed to evaluate the impact on the physicochemical properties and the in vitro cytotoxicity profile of transfersomes composed of single or mixed EAs with opposing HLB values. A pre-optimized formulation, using a qualityby-design approach, made of Tween ${ }^{\circledR} 80(\mathrm{HLB}=15)$ was the starting point for this work (13). The present study continued with EAs replaced and/or mixed with $\operatorname{Span}^{\circledR} 80(\mathrm{HLB}=4.3)$. The bioactive molecule chosen to be encapsulated in the prepared transfersomes was caffeine, as it has been widely used as hydrophilic model compound in dermal safety studies (14). Moreover, its antioxidant and lipolytic activities support its extensive use in cutaneous formulations (15). To our knowledge, this is the first report on the physicochemical characterization and in vitro cytotoxicity assessment of transfersomes prepared with mixed EAs with opposing HLB values to load caffeine.
$(4,5)$. De facto, este parâmetro deve ser otimizado, de preferência por estratégias de quality-by-design, uma vez que quantidades excessivas de EA $(>15 \%)$ podem causar transições vesícula-micela (11). Além disso, a seleção do EA para preparar transferossomas é também um fator-chave para modular o tamanho da vesícula e a sua eficiência de encapsulação. Considerando os tensioativos não-iónicos, os compostos hidrofílicos podem ser encapsulados de forma mais eficiente utilizando tensioativos com elevado equilíbrio hidrofílico-lipofílico (HLB), enquanto os tensioativos com valores baixos de HLB parecem encapsular melhor moléculas lipofílicas (5). Além disso, quanto mais baixo o HLB dos tensioativos não-iónicos, maior será o tamanho da vesícula (9) e menor a sua deformabilidade (10), o que pode reduzir a permeação cutânea do composto veiculado. No conjunto, estes dados indicam que a modulação dos valores de HLB através da utilização de misturas de EAs pode ser uma estratégia valiosa para otimizar o desempenho dos transferossomas.

Neste contexto, este estudo preliminar tem como objetivo avaliar o impacto nas propriedades físicoquímicas e no perfil de citotoxicidade in vitro de transferossomas constituídos por EAs únicos ou mistos, com valores opostos de HLB. Uma formulação préotimizada, utilizando uma abordagem de quality-bydesign, constituída por Tween $^{\circledR} 80(\mathrm{HLB}=15)$ foi o ponto de partida para este trabalho (13), sendo o EA substituído e/ou misturado com $\operatorname{Span}^{\circledR} 80(\mathrm{HLB}=4,3)$ no presente estudo. A cafeína foi o composto bioativo escolhido para ser encapsulado nos transferossomas preparados, uma vez que tem sido amplamente utilizada como composto modelo hidrofílico em estudos de segurança dérmica (14). Mais ainda, as propriedades antioxidantes e lipolíticas da cafeína apoiam o seu uso extensivo em formulações para aplicação cutânea (15). Segundo temos conhecimento, este é o primeiro trabalho quanto à caracterização físico-química e à avaliação in vitro da citotoxicidade de transferossomas constituídos por EAs mistos com valores de HLB opostos para veicular a cafeína. 


\section{Materials and Methods}

\section{Materials}

Caffeine was obtained from Fagron Ibérica (Terrassa, Spain). Lecithin from soybean was purchased from Alfa Aesar (Kandel, Germany). Tween ${ }^{\circledR} 80$, potassium chloride, monobasic potassium phosphate, MTT (3-(4,5-dimethylthiazol-2-yl)-2,5-diphenyltetrazolium bromide) and trypsin were obtained from Sigma-Aldrich (Saint Louis, MO, USA). Span ${ }^{\circledR} 80$ and DMSO (dimethyl sulfoxide) were purchased from Merck (Darmstadt, Germany). Chloroform and disodium hydrogen phosphate dihydrate were purchased from Scharlab S.L. (Sentmenat, Spain). Methanol was purchased from Carlo Erba Reagenti SpA (Rodano, Italy). Sephadex ${ }^{\mathrm{TM}}$ G-25 medium was obtained from GE Healthcare Biosciences AB (Uppsala, Sweden). Sodium chloride was purchased from José M. Vaz Pereira SA (Benavente, Portugal). Low glucose DMEM (Dulbecco's Modified Eagle Medium) and FBS (fetal bovine serum) were purchased from Biowest (Nuaillé, France). Penicillinstreptomycin (pen/strep) was obtained from Grisp (Porto, Portugal). Phosphate-buffered saline (PBS) at $\mathrm{pH} 7.4$ was prepared as previously described (13).

\section{Preparation of transfersomes}

Transfersomes were prepared using the lipid-film hydration method followed by sonication, as previously described (13). Seven formulations were prepared, with or without caffeine, consisting of lecithin (PC) and Tween ${ }^{\circledR} 80$ and/or Span ${ }^{\circledR} 80$ as EA, according to Table 1.

EA:PC films were obtained by dissolving the materials in chloroform:methanol $(3: 1, \mathrm{v} / \mathrm{v})$ before solvent evaporation using a rotary evaporator (Model VV2000/ VB2000, Heidolph Instruments, Schwabach, Germany) at $40{ }^{\circ} \mathrm{C}, 90 \mathrm{rpm}$, for $30 \mathrm{~min}$. Traces of organic solvents were removed under vacuum for $2 \mathrm{~h}$. The transfersomal formulations were prepared by hydrating the EA:PC films with PBS buffer or a $1 \%(\mathrm{w} / \mathrm{v})$ caffeine buffered solution with vigorous vortexing. Finally, the formulations were sonicated (model Q125 sonicator, QSonica Sonicators, Newtown, CT, USA) at 50\% amplitude for $10 \mathrm{~min}$.

\section{Materiais e Métodos}

\section{Materiais}

A cafeína foi obtida a partir da Fagron Ibérica (Terrassa, Espanha), enquanto a lecitina da soja foi adquirida na Alfa Aesar (Kandel, Alemanha). O Tween ${ }^{\circledR}$ 80, o cloreto de potássio, o fosfato monobásico de potássio, o MTT (brometo de 3-(4,5-dimetiltiazol-2-il)-2,5difeniltetrazólio) e a tripsina foram obtidos a partir da Sigma-Aldrich (Saint Louis, MO, EUA). O Span ${ }^{\circledR}$ 80 e o DMSO (dimetilsulfóxido) foram adquiridos na Merck (Darmstadt, Alemanha). O clorofórmio e o hidrogenofosfato dissódico di-hidratado foram adquiridos na Scharlab S.L. (Sentmenat, Espanha), enquanto o metanol foi obtido a partir da Carlo Erba Reagenti SpA (Rodano, Itália). O Sephadex ${ }^{\mathrm{TM}}$ G-25 médio foi adquirido na GE Healthcare Bio-sciences AB (Uppsala, Suécia). O cloreto de sódio foi obtido a partir da José M. Vaz Pereira, SA (Benavente, Portugal). O DMEM (Dulbecco's Modified Eagle Medium) e o FBS (soro bovino fetal) foram adquiridos na Biowest (Nuaillé, França). A penicilina-estreptomicina (pen/ strep) foi obtida a partir da Grisp (Porto, Portugal). O tampão fosfato (PBS) a $\mathrm{pH} 7,4$ foi preparado como descrito anteriormente (13).

\section{Preparação dos transferossomas}

O método de hidratação do filme lipídico seguido de sonicação foi utilizado para preparar transferossomas, tal como descrito anteriormente (13). Foram preparadas sete formulações, com ou sem cafeína, constituídas por lecitina (PC) e Tween ${ }^{\circledR} 80$ e/ou Span ${ }^{\circledR} 80$ como EAs, tal como descrito na Tabela 1.

Os filmes compostos por EA e PC foram obtidos através da dissolução destes compostos em clorofórmio:metanol $(3: 1, \quad v / v)$ seguida da evaporação dos solventes utilizando o evaporador rotativo VV2000/VB2000 (Heidolph Instruments, Schwabach, Alemanha) a 40 ${ }^{\circ} \mathrm{C}$, $90 \mathrm{rpm}$, durante $30 \mathrm{~min}$. Os vestígios de solventes orgânicos foram ainda removidos sob vácuo durante $2 \mathrm{~h}$. As formulações transferossomais foram obtidas, de seguida, através da hidratação dos filmes obtidos anteriormente com tampão PBS ou uma solução tamponada de cafeína $(1 \%(\mathrm{~m} / \mathrm{v}))$, seguida de agitação vigorosa. Por fim, as formulações foram sonicadas durante $10 \mathrm{~min}$ (50\% de amplitude), utilizando o sonicador Q125 (QSonica Sonicators, Newtown, CT, EUA). 
Table 1 - Qualitative and quantitative composition of transfersomal formulations.

Tabela 1 - Composição qualitativa e quantitativa das formulações transferossomais.

\begin{tabular}{cccccc}
\hline $\begin{array}{c}\text { Formulation / } \\
\text { Formulação }\end{array}$ & $\begin{array}{c}\text { PC } \\
(\% \mathrm{w} / \mathrm{v})\end{array}$ & $\begin{array}{c}\mathbf{T w e e n}^{\circledR} \\
\mathbf{8 0 : P C} \\
(\mathrm{w} / \mathrm{w})\end{array}$ & $\begin{array}{c}\text { Span }^{\circledR} \\
\mathbf{8 0 : P C} \\
(\mathrm{w} / \mathrm{w})\end{array}$ & $\begin{array}{c}\text { HLB of } \\
\text { EA }\end{array}$ & $\begin{array}{c}\text { CAF } \\
(\% \mathrm{w} / \mathrm{v})\end{array}$ \\
\hline $\mathbf{1 U}$ & 1 & $15: 85$ & - & 15 & - \\
$\mathbf{1 L}$ & 1 & $15: 85$ & - & 15 & 1 \\
$\mathbf{2 U}$ & 1 & $7.5: 85$ & $7.5: 85$ & 9.65 & - \\
$\mathbf{2} \mathbf{L}$ & 1 & $7.5: 85$ & $7.5: 85$ & 9.65 & 1 \\
$\mathbf{3 U}$ & 1 & - & $15: 85$ & 4.3 & - \\
3L & 1 & - & $15: 85$ & 4.3 & 1 \\
PC & 1 & - & - & - & - \\
\hline
\end{tabular}

PC: lecithin / lecitina; HLB: hydrophilic-lipophilic balance / equilíbrio hidrofílico-lipofílico; EA: edge activators / ativador(es) de superfície; CAF: caffeine / cafeína; L: loaded with caffeine/presença de cafeína; U: unloaded with caffeine/ausência de cafeína.

\section{Characterization of transfersomes}

Immediately after preparation, the transfersomes were characterized according to their hydrodynamic diameter $\left(\mathrm{D}_{\mathrm{h}}\right)$, polydispersity index $(\mathrm{PDI})$, encapsulation efficiency (EE), and loading capacity (LC). The results were obtained from triplicate analysis of each formulation and reported as mean \pm standard deviation (SD).

The $\mathrm{D}_{\mathrm{h}}$ and PDI values of transfersomal formulations, diluted 1:20 with distilled water, were determined at room temperature $\left(25 \pm 2{ }^{\circ} \mathrm{C}\right)$ by dynamic light scattering measurements (Delsa ${ }^{\mathrm{TM}}$ Nano $\mathrm{C}$ equipment, Beckman Coulter, Inc., CA, USA).

The EE and LC of the transfersomes were analyzed using an indirect approach by the determination of the nonencapsulated fraction of caffeine using size exclusion chromatography, as previously described (13), with some modifications. A $500 \mu \mathrm{l}$ aliquot of each formulation was applied to prepared Sephadex ${ }^{\mathrm{TM}}$ G-25 medium (stationary phase). Following elution of the caffeine-encapsulated fractions (confirmed by visual analysis) with PBS (mobile phase), the nonencapsulated fraction of caffeine was collected for $30 \mathrm{~min}$ and the collected volume was recorded for accurate calculation of the dilution factor (collected volume $/ 500 \mu \mathrm{l}$ ).

\section{Caraterização dos transferossomas}

Imediatamente após a preparação, os transferossomas foram caracterizados quanto ao seu diâmetro hidrodinâmico $\left(\mathrm{D}_{\mathrm{h}}\right)$, índice de polidispersão (PDI), eficiência de encapsulação (EE), e capacidade de carga (LC). Os resultados foram obtidos a partir da análise em triplicado de cada formulação e estão representados como média \pm desvio padrão (SD).

Os valores de $\mathrm{D}_{\mathrm{h}}$ e PDI das formulações transferossomais foram determinados por medições espectroscopia de correlação fotónica (equipamento Delsa ${ }^{\mathrm{TM}}$ Nano C, Beckman Coulter, Inc., CA, EUA) à temperatura ambiente $\left(25 \pm 2{ }^{\circ} \mathrm{C}\right)$. Antes das medições, cada formulação foi diluída com água destilada (1:20).

A EE e LC dos transferossomas foram analisadas utilizando uma abordagem indireta, através da determinação da fração não encapsulada de cafeína utilizando a cromatografia de exclusão de tamanho, tal como anteriormente descrito com algumas modificações (13). O Sephadex ${ }^{\mathrm{TM}} \mathrm{G}-25$ médio foi utilizado como fase estacionária e o PBS como fase móvel para eludir uma alíquota de $500 \mu \mathrm{l}$ de cada formulação. Após a eluição das frações com cafeína encapsulada (confirmada por análise visual), a fração não encapsulada de cafeína foi recolhida durante 30 min e o volume recolhido foi registado para ser considerado no cálculo do fator de diluição (volume recolhido/500 $\mu \mathrm{l}$ ). 
The concentration of caffeine in the nonencapsulated fraction was determined by UV-Vis spectroscopy (Evolution ${ }^{\circledR}$ 300, Thermo Scientific, Hertfordshire, England) using a calibration curve and considering the dilution factor. Six solutions of caffeine over a concentration range of $0-25 \mu \mathrm{g} / \mathrm{ml}$ were prepared in PBS to establish the concentration curve. The absorption spectrum $(200-350 \mathrm{~nm}, 600 \mathrm{~nm} / \mathrm{min})$ of each solution was likewise obtained by UV/Vis spectroscopy. The calibration curve of caffeine $(y=0.0539 x-0.0077$, $\left.\mathrm{R}^{2}=0.9991\right)$ was then determined considering the absorbance of caffeine at the absorption maximum wavelength $(273 \mathrm{~nm})$ as a function of caffeine concentration $(\mu \mathrm{g} / \mathrm{ml})$.

EE and LC values, represented as percentage (\%), were calculated according to Equations 1 and 2, respectively:

$$
\begin{aligned}
& E E=\frac{C_{T}-C_{N E}}{C_{T}} \times 100 \\
& L C=\frac{C_{T}-C_{N E}}{C_{L}} \times 100
\end{aligned}
$$

where $C_{T}$ represents the total concentration of caffeine, $C_{N E}$ the concentration of nonencapsulated caffeine, and $C_{L}$ the total concentration of PC.

\section{Stability studies}

The formulations were stored at $5 \pm 2{ }^{\circ} \mathrm{C}$ for two months. The stability of $\mathrm{D}_{\mathrm{h}}$ and PDI values of stored transfersomes was evaluated at different time points (Day 23, 30, and 60) and compared to the values obtained with freshly made formulations (Day 0). These parameters were evaluated as described above.

\section{In vitro cytotoxicity assessment}

Cell viability studies were performed using the HaCat cell line, representative of human keratinocytes, which was cultured in low glucose DMEM, supplemented with $10 \%$ FBS and $1 \%$ pen/strep and was kept at $37{ }^{\circ} \mathrm{C}$ under a humidified air atmosphere with $5 \% \mathrm{CO}_{2}$.

The MTT reduction method was used to assess the cytotoxicity of the transfersomal formulations, in line with previous publications $(16,17)$. Briefly, HaCat cells were seeded $\left(6 \times 10^{3}\right.$ cells/well, $200 \mu 1$ of supplemented DMEM) in 96-well plates and were allowed to incubate for $24 \mathrm{~h}$. After that, cells were treated for $24 \mathrm{~h}$ with a)
A concentração de cafeína na fração não encapsulada foi determinada por espectroscopia UV-Vis (Evolution ${ }^{\circledR}$ 300, Thermo Scientific, Hertfordshire, Inglaterra), utilizando um método de curva de calibração e considerando o fator de diluição. Para determinar a curva de calibração da cafeína, seis soluções de cafeína em PBS foram preparadas numa gama de concentração de 0-25 $\mu \mathrm{g} / \mathrm{ml}$. O espectro de absorção $(200-350$ nm, 600 $\mathrm{nm} / \mathrm{min}$ ) de cada solução foi obtido por espectroscopia UV/Vis (espectrofotómetro Evolution ${ }^{\circledR}$ 300, Thermo Scientific, Hertfordshire, Inglaterra). A curva de calibração da cafeína ( $\left.\mathrm{y}=0,0539 \mathrm{x}-0,0077, \mathrm{R}^{2}=0,9991\right)$ foi então determinada, considerando a absorvência da cafeína no comprimento de onda máximo de absorção (273 nm) em função da concentração de cafeína ( $\mu \mathrm{g} /$ $\mathrm{ml})$.

Os valores de EE e LC, apresentados em percentagem (\%), foram calculados de acordo com as Equações $1 \mathrm{e}$ 2, respetivamente:

$$
\begin{aligned}
& E E=\frac{C_{T}-C_{N E}}{C_{T}} \times 100 \\
& L C=\frac{C_{T}-C_{N E}}{C_{L}} \times 100
\end{aligned}
$$

onde $\mathrm{C}_{\mathrm{T}}$ representa a concentração total de cafeína, $\mathrm{C}_{\mathrm{NE}}$ a concentração de cafeína não-encapsulada, e $\mathrm{C}_{\mathrm{L}}$ a concentração total de PC.

\section{Estudos de estabilidade}

As formulações foram armazenadas a $5 \pm 2{ }^{\circ} \mathrm{C}$ durante 2 meses e a estabilidade dos valores de $\mathrm{D}_{\mathrm{h}}$ e PDI dos transferossomas foi avaliada em diferentes momentos (Dia 23, 30 e 60) em comparação com os valores obtidos com as formulações no dia da preparação (Dia 0). Estes parâmetros foram avaliados tal como especificado na seção anterior.

\section{Avaliação in vitro da citotoxicidade}

Foram efetuados estudos de viabilidade celular utilizando a linha celular HaCat, representativa de queratinócitos humanos, que foi cultivada em DMEM, suplementada com $10 \%$ FBS e $1 \%$ pen/strep e mantida a $37{ }^{\circ} \mathrm{C}$ sob atmosfera de ar humidificado com $5 \%$ de $\mathrm{CO}_{2}$.

O método de redução MTT foi utilizado para avaliar a citotoxicidade das formulações transferossomais, tal como especificado em publicações anteriores $(16,17)$. Em resumo, as células HaCat foram semeadas $\left(6 \times 10^{3}\right.$ 
control solutions, namely PBS or DMSO $(5 \%, \mathrm{v} / \mathrm{v})$ as negative and positive control (respectively); b) each transfersomal formulation diluted 1:100 and 1:200 in PBS, corresponding to caffeine concentrations of 515.7 and $257.9 \mu \mathrm{M}$ (respectively); and c) buffered solutions of each individual component of the formulations, considering the quantitative composition of the formulations and the applied dilution factors. Afterwards, cells were washed with PBS and $200 \mu$ of MTT solutions $(0.5 \mathrm{mg} / \mathrm{ml})$ in supplemented DMEM was added to each well, followed by an incubation period of $4 \mathrm{~h}$. After another wash with PBS, $200 \mu \mathrm{l}$ of DMSO were added to each well to dissolve the formazan crystals. Finally, the absorption values, at $595 \mathrm{~nm}$, of each well were acquired using a microplate photometer (Thermo Scientific ${ }^{\mathrm{TM}}$ Multiskan $^{\mathrm{TM}} \mathrm{FC}$ ). The absorption of PBS-treated cells was used as reference, representing $100 \%$ cell viability. Results were obtained from quadruplicates of each condition in two independent assays and are presented as mean $\pm \mathrm{SD}$.

\section{Statistical analysis}

All data were statistically analyzed using GraphPad Prism 8.0.2 (GraphPad Software, San Diego, California, USA). Physicochemical characterization and cell viability data were analyzed through unilateral variance analysis test (One-way ANOVA), followed by Turkey's or Dunnett's multiple comparison (respectively). Results regarding the stability studies were analyzed through bilateral variance analysis test (Two-way ANOVA), followed by Tukey's multiple comparison. Differences were considered as statistically significant for $p$ values under $0.05(p<0.05)$.

\section{Results and Discussion}

\section{Characterization of transfersomal formulations}

The impact of using single or mixed EAs, namely Tween ${ }^{\circledR}$ 80 (1), Tween ${ }^{\circledR} 80: \operatorname{Span}^{\circledR} 80$ 1:1 (2) or $\operatorname{Span}^{\circledR} 80$ (3), to prepare transfersomes in the absence $(\mathrm{U})$ and presence of caffeine (L) was evaluated in terms of $\mathrm{D}_{\mathrm{h}}, \mathrm{PDI}, \mathrm{EE}$, and LC. The results are summarised in Figure 1.

Regarding $D_{h}$ data, all formulations have average diameters between 93 and $111 \mathrm{~nm}$, which is a suitable range for transcutaneous application. In fact, previous células/poço, $200 \mu \mathrm{l}$ de DMEM suplementado) em placas de 96 poços e foram incubadas durante 24 h. Depois disso, as células foram tratadas durante $24 \mathrm{~h}$ com: a) soluções controlo, nomeadamente PBS ou DMSO $(5 \%, \mathrm{v} / \mathrm{v})$ como controlo negativo e positivo (respetivamente); b) cada formulação transferossomal após diluições de 1:100 e 1:200 em PBS, correspondendo a concentrações de cafeína de 515,7 e $257,9 \mu \mathrm{M}$ (respetivamente); e c) soluções tamponadas de cada componente individual das formulações, considerando a composição quantitativa das formulações e os fatores de diluição aplicados. Posteriormente, as células foram lavadas com PBS e adicionaram-se $200 \mu 1$ de solução de MTT $(0,5 \mathrm{mg} /$ $\mathrm{ml}$ ) em DMEM suplementado a cada poço, seguido de um período de incubação de $4 \mathrm{~h}$. Após outra etapa de lavagem com PBS, adicionaram-se $200 \mu 1$ de DMSO a cada poço para dissolver os cristais de formazan. Finalmente, os valores de absorvência, a $595 \mathrm{~nm}$, foram analisados em cada poço utilizando o fotómetro de microplaca Thermo Scientific ${ }^{\mathrm{TM}}$ Multiskan $^{\mathrm{TM}}$ FC. A absorvência obtida com as células tratadas com PBS foi utilizada como referência, representando $100 \%$ de viabilidade celular. Os resultados foram obtidos a partir de quadruplicados de cada condição, em dois ensaios independentes, e são apresentados como média \pm SD.

\section{Análise estatística}

Todos os dados foram analisados estatisticamente usando o GraphPad Prism 8.0.2 (GraphPad Software, San Diego, Califórnia EUA). Os dados de caraterização físico-química e de viabilidade celular foram analisados através de teste de análise de variância unilateral (Oneway ANOVA), seguido por comparação múltipla de Turkey ou Dunnett (respetivamente). Os resultados relativos aos estudos de estabilidade foram analisados através do teste de análise de variância bilateral (ANOVA bidirecional), seguido de comparação múltipla de Tukey. As diferenças foram consideradas como estatisticamente significativas para valores de $p$ inferiores a $0,05(p<0,05)$.

\section{Resultados e Discussão}

\section{Caraterização das formulações transferossomais}

O impacto da utilização de EAs únicos ou mistos, nomeadamente Tween ${ }^{\circledR} 80$ (1), Tween ${ }^{\circledR} 80: \operatorname{Span}^{\circledR} 80$ 1:1 (2) ou $\operatorname{Span}^{\circledR} 80$ (3), para preparar transferossomas na ausência (U) e presença (L) de cafeína foi avaliado 
studies indicate that transfersomes smaller than $300 \mathrm{~nm}$ are highly deformable and able to penetrate to deeper layers of the skin (4) and that the smaller the vesicle size, the greater its penetration into the skin $(8,18)$.

As shown in Figure 1a, the incorporation of caffeine did not change the size of transfersomes regardless of the type of EA used $(1 \mathrm{U}-1 \mathrm{~L}, 2 \mathrm{U}-2 \mathrm{~L}$, and $3 \mathrm{U}-$ 3L comparisons). On the other hand, the size of the transfersomes varied according to the type of EA used both in the absence and presence of caffeine. In fact, using Tween ${ }^{\circledR} 80$ as EA (1U or 1L) yielded smaller transfersomes than those prepared with the mixture of Tween $^{\circledR} 80$ and $\operatorname{Span}^{\circledR} 80$ (2U or 2L) or with $\operatorname{Span}^{\circledR} 80$ alone $(3 \mathrm{U}$ or $3 \mathrm{~L})$. The significant increase of $\mathrm{D}_{\mathrm{h}}$ values caused by the incorporation of $\operatorname{Span}^{\circledR} 80$ was also observed in comparison with the PC formulation (2U$\mathrm{PC}$ and 3U-PC comparisons), prepared in the absence of EAs. These results are in agreement with previously published results (9), as the increase in vesicle size may be related to the affinity of the surfactant for the phospholipid, with $\operatorname{Span}^{\circledR} 80 \quad(\mathrm{HLB}=4.3)$ having a higher affinity for $\mathrm{PC}$, as it is more hydrophobic than Tween $^{\circledR} 80(\mathrm{HLB}=15)(10)$. em termos de $\mathrm{D}_{\mathrm{h}}$, PDI, EE e LC. Os resultados obtidos estão apresentados na Figura 1.

Em relação aos dados $D_{h}$, todas as formulações apresentaram diâmetros médios entre 93 e $111 \mathrm{~nm}$, o que as torna adequadas para aplicação transcutânea. De facto, estudos anteriores indicam que transferossomas inferiores a $300 \mathrm{~nm}$ são altamente deformáveis e capazes de penetrar em camadas mais profundas da pele (4) e que quanto menor o tamanho da vesícula, maior a sua penetração na pele $(8,18)$.

Através da análise da Figura 1a, verifica-se que a incorporação de cafeína não alterou o tamanho dos transferossomas, independentemente do tipo de EA utilizado (comparações 1U-1L, 2U-2L, e 3U-3L). Por outro lado, o tamanho dos transferossomas variou de acordo com o tipo de EA utilizado, tanto na ausência como na presença de cafeína. De facto, a utilização de Tween ${ }^{\circledR} 80$ como EA (1U ou 1L) deu origem a transferossomas menores do que os preparados com a mistura de Tween ${ }^{\circledR} 80$ e $\operatorname{Span}^{\circledR} 80$ (2U ou 2L) ou apenas com $\operatorname{Span}^{\circledR} 80$ (3U ou 3L). O aumento significativo dos valores de $\mathrm{D}_{\mathrm{h}}$ causado pela incorporação de $\operatorname{Span}^{\circledR} 80$ também foi observado em comparação com a formulação PC (comparações 2U-PC e 3U-PC), preparada na ausência de EAs. Estes resultados estão de acordo com a literatura (9), uma vez que o aumento
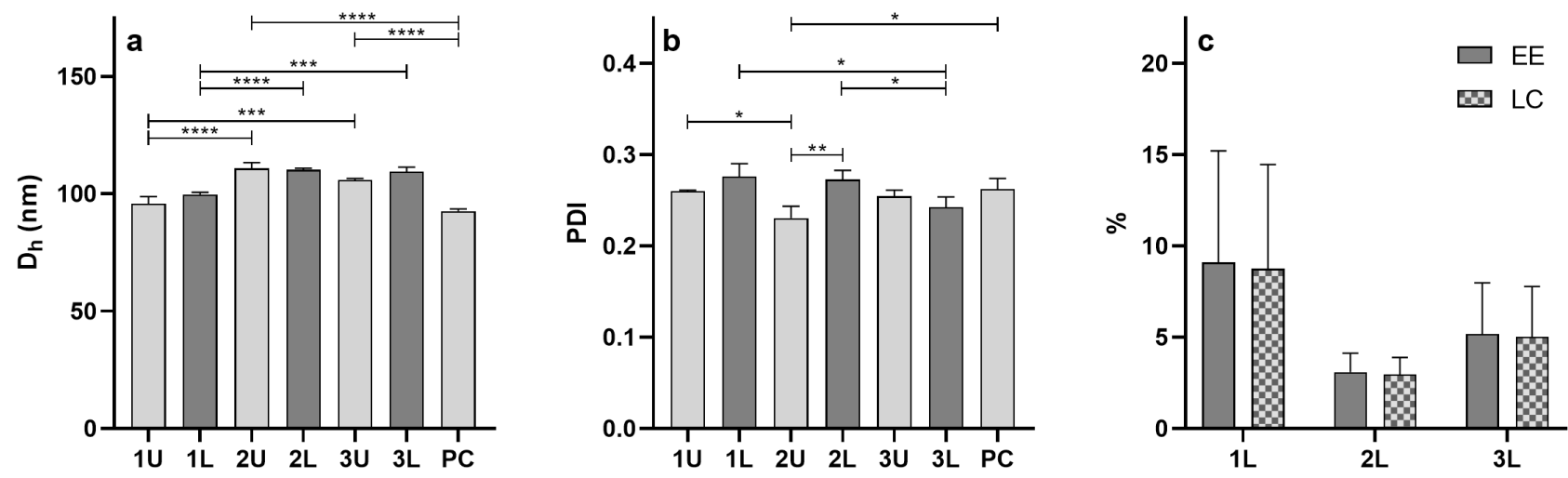

Figure 1 - Physicochemical characterization of transfersomal formulations, in the absence (U) and presence (L) of caffeine, in terms of (a) hydrodynamic diameter $\left(\mathrm{D}_{\mathrm{h}}\right)$, (b) polydispersity index (PDI), and (c) encapsulation efficiency (EE) and loading capacity (LC).

Figura 1 - Caracterização físico-química das formulações transferossomais, na ausência (U) e na presença (L) de cafeína, em termos de (a) diâmetro hidrodinâmico $\left(\mathrm{D}_{\mathrm{h}}\right)$, (b) índice de polidispersão (PDI), e (c) eficiência de encapsulação (EE) e capacidade de carga (LC).

The edge activators (EA) used in each formulation are identified as 1 (Tween ${ }^{\circledR} 80$ ), 2 (Tween ${ }^{\circledR} 80$ and Span ${ }^{\circledR} 80$ ), 3 (Span ${ }^{\circledR}$ 80), and PC (no EA or caffeine). All data are presented as mean $\pm S D(n=3)$ and the data sets with significant differences are identified with $* \mathrm{p}<0.05,{ }^{* *}<<0.01$, $* * * \mathrm{p}<0.001$ or $* * * * \mathrm{p}<0.0001$.

O(s) ativador(es) de superfície (EA) utilizados em cada formulação estão identificados por 1 (Tween $\left.{ }^{\circledR} 80\right)$, 2 (Tween ${ }^{\circledR} 80$ e Span $\left.{ }^{\circledR} 80\right)$, 3 (Span $\left.{ }^{\circledR} 80\right)$ e PC (sem incorporação de EA e cafeína). Todos os dados estão representados como média \pm SD ( $\left.n=3\right)$ e os conjuntos de dados com diferenças significativas estão identificados com $* \mathrm{p}<0,05, * * \mathrm{p}<0,01, * * *_{\mathrm{p}}<0,001$ ou $* * * * \mathrm{p}<0,0001$. 
The PDI values of transfersomal formulations was also evaluated (Figure 1b), and the average PDI values ranged between 0.23 and 0.28 . It is noteworthy that smaller values of PDI in the absence of caffeine were obtained by preparing transfersomes with a mixture of Tween ${ }^{\circledR} 80$ and $\operatorname{Span}^{\circledR} 80(\mathrm{PDI}(2 \mathrm{U})=0.23 \pm 0.01)$. In contrast, in the presence of caffeine, the uniformity of size distribution was superior for transfersomes prepared with $\operatorname{Span}^{\circledR} 80$ (PDI(3L) $\left.=0.24 \pm 0.01\right)$. Despite the small differences found in PDI values, the gathered data indicate that all transfersomal formulations are composed of monodisperse populations of vesicles, as PDI values lower than 0.3 are considered acceptable regarding the uniformity of vesicle size (19).

Concerning EE and LC of the prepared transfersomes, the average values ranged between $3 \%$ and $9 \%$, with no significant differences according to the type of EAs used (Figure 1c). These results suggest that the amount of caffeine added exceeded the carrying capacity of the vesicles, so a decrease in the caffeine concentration and/or an increase in the concentration of excipients could potentially increase the EE and LC of these transfersomes in future studies. Due to the hydrophilic character of caffeine, its encapsulation will likely depend on the water content of the core of vesicles, which is directly related to number of vesicles in solution, thereby depending on the phospholipid concentration used to prepare the formulations (20). This hypothesis is in line with the results of Colletier et al. (21) who reported improvement of the EE of acetylcholinesterase by enhancing the phospholipid concentration used in liposome formulations.

\section{Storage stability of transfersomal formulations}

The physicochemical stability of the transfersomal formulations stored at $5 \pm 2{ }^{\circ} \mathrm{C}$ was evaluated for two months by analyzing $\mathrm{D}_{h}$ and PDI of the transfersomes as a function of time (Figure 2). It is noteworthy that the properties of the formulations remained stable for one month. However, the diameter of the transfersomes significantly increased after two months in all cases, with a concomitant loss of vesicle size homogeneity (increased PDI) in the case of the $1 \mathrm{U}, 1 \mathrm{~L}$, and PC formulations. The increase in $D_{h}$ values over time may be related to vesicle aggregation, that is more likely do tamanho da vesícula pode estar relacionado com a afinidade do tensoativo para o fosfolípido, apresentando o $\operatorname{Span}^{\circledR} 80(\mathrm{HLB}=4,3)$ uma afinidade maior para o PC, uma vez que é mais hidrofóbico que o Tween ${ }^{\circledR} 80$ $(\mathrm{HLB}=15)(10)$.

Os valores de PDI das formulações transferossomais também foram avaliados (Figura 1b) e os valores médios de PDI variaram entre 0,23 e 0,28 . É de notar que foram obtidos valores mais baixos de PDI na ausência de cafeína quando se prepararam transferossomas com uma mistura de Tween ${ }^{\circledR} 80$ e $\operatorname{Span}^{\circledR} 80 \quad(\mathrm{PDI}(2 \mathrm{U})=0,23 \pm 0,01)$. Em contraste, na presença de cafeína, a uniformidade da distribuição do tamanho foi superior para transferossomas preparados com $\operatorname{Span}^{\circledR} 80 \quad(\mathrm{PDI}(3 \mathrm{~L})=0,24 \pm 0,01)$. Apesar das pequenas diferenças encontradas nos valores de PDI, os dados obtidos indicam que todas as formulações de transferossomas são compostas por populações monodispersas de vesículas, uma vez que valores de PDI inferiores a 0,3 são considerados aceitáveis relativamente à uniformidade do tamanho de vesículas (19).

Relativamente ao EE e LC dos transferossomas preparados, os valores médios variaram entre 3\% e $9 \%$, sem diferenças significativas de acordo com o tipo de EAs utilizado(s) (Figura 1c). Estes resultados sugerem que a quantidade de cafeína adicionada excedeu a capacidade de carga das vesículas, pelo que uma diminuição na concentração de cafeína e/ou um aumento na concentração de excipientes poderia possivelmente aumentar o EE e LC destes transferossomas em estudos futuros. Devido ao carácter hidrofílico da cafeína, a sua encapsulação dependerá provavelmente do teor de água do núcleo das vesículas, que está diretamente relacionado com o número de vesículas em solução, dependendo assim da concentração de fosfolípidos utilizada para preparar as formulações (20). Esta hipótese está de acordo com os resultados de Colletier et al. (21) que foram capazes de melhorar o EE da acetilcolinesterase através do aumento da concentração de fosfolípidos utilizada nas formulações de lipossomas.

\section{Estabilidade das formulações transferossomais longo do armazenamento} ao

A estabilidade físico-química das formulações transferossomais armazenadas a $5 \pm 2{ }^{\circ} \mathrm{C}$ foi avaliada durante dois meses através da análise de $\mathrm{D}_{\mathrm{h}}$ e PDI dos transferossomas em função do tempo (Figura 2). É de salientar que as propriedades das formulações permaneceram estáveis durante um mês. No entanto, o diâmetro dos transferossomas aumentou 
to occur when the surface charge of vesicles is close to neutrality $(4,22)$. This may be the case in our study, since the main component of the prepared transfersomes is lecithin, a zwitterionic phospholipid. Thus, it would be of interest to evaluate the zeta potential of these vesicular systems in future studies to better understand the role of superficial charge on the aggregation phenomenon of these formulations. significativamente após 2 meses em todos os casos, com uma concomitante perda de homogeneidade do tamanho das vesículas (aumento do PDI) no caso das formulações $1 \mathrm{U}, 1 \mathrm{~L}$ e PC. O aumento dos valores de $\mathrm{D}_{\mathrm{h}}$ ao longo do tempo pode estar relacionado com a agregação de vesículas, o que é mais provável quando a carga superficial das vesículas está próxima da neutralidade $(4,22)$, o que pode ser o caso, uma vez que o componente principal dos transferossomas preparados é a lecitina, um fosfolípido zwitteriónico. Assim, seria interessante avaliar o potencial zeta destes sistemas vesiculares em estudos futuros para melhor compreender o papel da carga superficial das vesículas no fenómeno de agregação destas formulações.
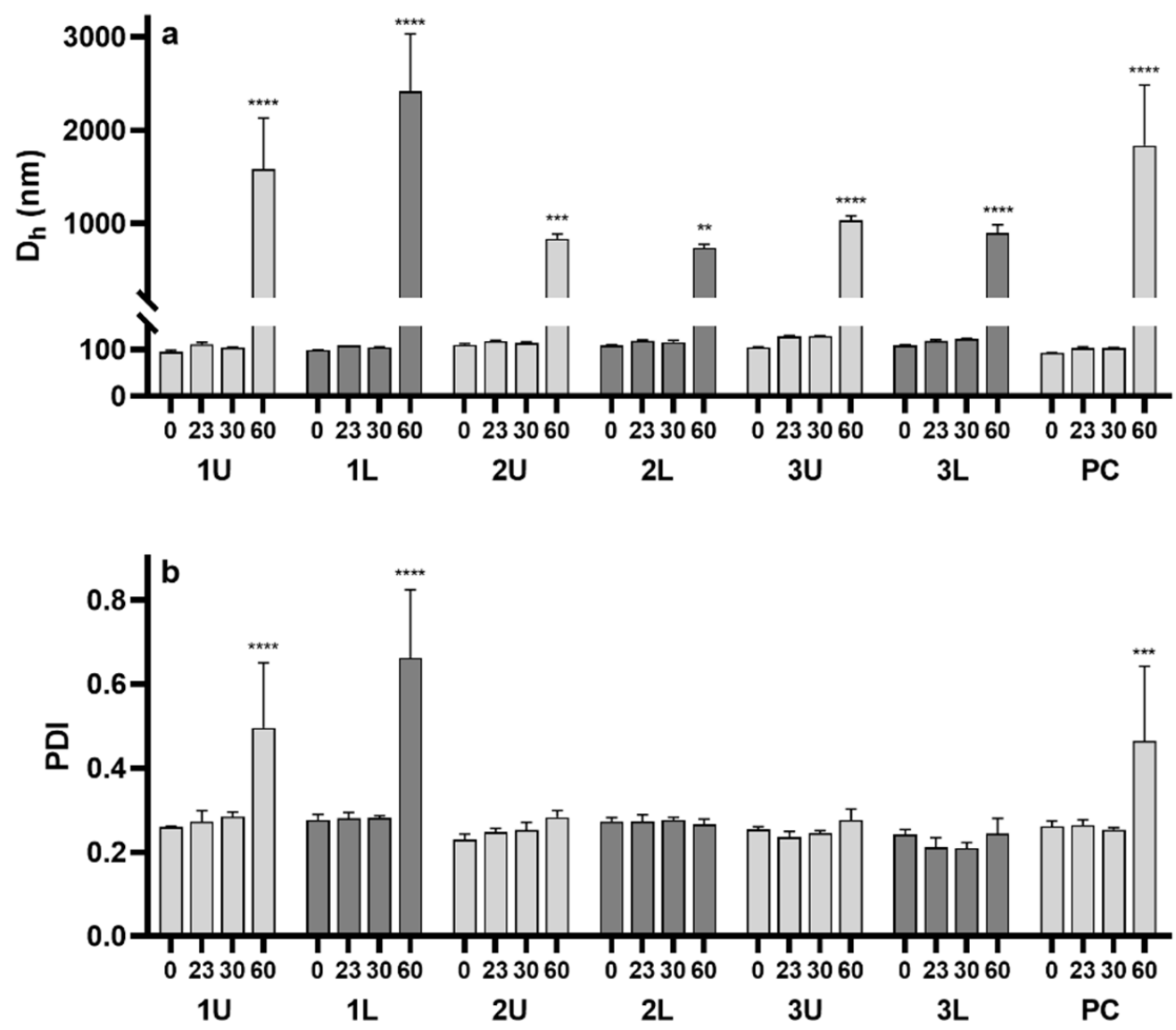

Figure 2 - Stability of transfersomal formulations, in the absence (U) and presence (L) of caffeine, in terms of (a) hydrodynamic diameter $\left(\mathrm{D}_{\mathrm{h}}\right)$ and $(\mathrm{b})$ polydispersity index $(\mathrm{PDI})$ as a function of time $(0,23,30$, and 60 days) of refrigerated storage.

Figura 2 - Estabilidade das formulações transferossomais, na ausência (U) e na presença (L) de cafeína, em termos de (a) diâmetro hidrodinâmico $\left(\mathrm{D}_{\mathrm{h}}\right)$ e (b) índice de polidispersão (PDI) em função do tempo (0, 23, 30 e 60 dias) sob armazenamento refrigerado.

The edge activators (EA) used in each formulation are identified as 1 (Tween $\left.{ }^{\circledR} 80\right), 2$ (Tween ${ }^{\circledR} 80$ and Span $\left.{ }^{\circledR} 80\right), 3$ (Span $\left.{ }^{\circledR} 80\right)$, and PC (no addition of EA or caffeine). All data were presented as mean $\pm S D(n=3)$ and the data sets with significant differences in relation to $t=0$ are identified with $* * \mathrm{p}<0.01$, *** $\mathrm{p}<0.001$ or $* * * * \mathrm{p}<0.0001$.

$O(s)$ ativador(es) de superfície (EA) utilizados em cada formulação estão identificados por 1 (Tween ${ }^{\circledR}$ 80), 2 (Tween ${ }^{\circledR} 80$ e Span $\left.{ }^{\circledR} 80\right)$, 3 (Span $\left.{ }^{\circledR} 80\right)$ e PC (sem incorporação de EA e cafeína). Todos os dados estão representados como média $\pm S D(n=3)$ e os conjuntos de dados com diferenças significativas em relação a $t=0$ estão identificados com $* * \mathrm{p}<0,01$, ***p $<0,001$ ou $* * * * \mathrm{p}<0,0001$. 
In vitro cytotoxicity assessment of transfersomal formulations

The cytotoxicity potential of transfersomal formulations prepared with single or mixed EAs, in the absence and presence of caffeine, was evaluated upon treatment of HaCat cells for $24 \mathrm{~h}$ using the MTT reduction method. The results collected for all formulations and all individual components of the formulations, after a 1:100 or 1:200 dilution in PBS, are presented in Figure 3. It is noteworthy that the treatment with the positive control (DMSO-treated cells) caused a significant reduction in cell viability compared to control cells (PBS-treated cells), as expected.

Human keratinocytes treated with encapsulated or nonencapsulated caffeine, at both concentrations under study $(515.7$ and $257.9 \mu \mathrm{M})$, did not show significant differences in cell viability when compared to control cells. These data are in agreement with previously reported studies showing that the treatment of HaCat cells with a caffeine concentration below $512 \mu \mathrm{M}$ for 24 $\mathrm{h}$ does not affect the cellular viability $(23,24)$.
Avaliação in vitro da citotoxicidade das formulações transferossomais

O potencial de citotoxicidade das formulações transferossomais preparadas com EAs únicos ou mistos, na ausência e na presença de cafeína, foi avaliado após tratamento de células HaCat durante $24 \mathrm{~h}$ através do método de redução MTT. Os resultados obtidos para todas as formulações e para todos os componentes individuais das formulações, após uma diluição de 1:100 ou 1:200 em PBS, estão apresentados na Figura 3. É de salientar que o tratamento com o controlo positivo (células tratadas com DMSO) causou uma redução significativa da viabilidade celular em comparação com o controlo negativo (células tratadas com PBS), tal como esperado.

Os queratinócitos humanos tratados com cafeína encapsulada ou não encapsulada, em ambas as concentrações em estudo (515,7 e 257,9 $\mu \mathrm{M})$, não apresentaram diferenças significativas quanto à viabilidade celular quando comparados com as células tratadas com PBS (controlo). Estes dados estão de

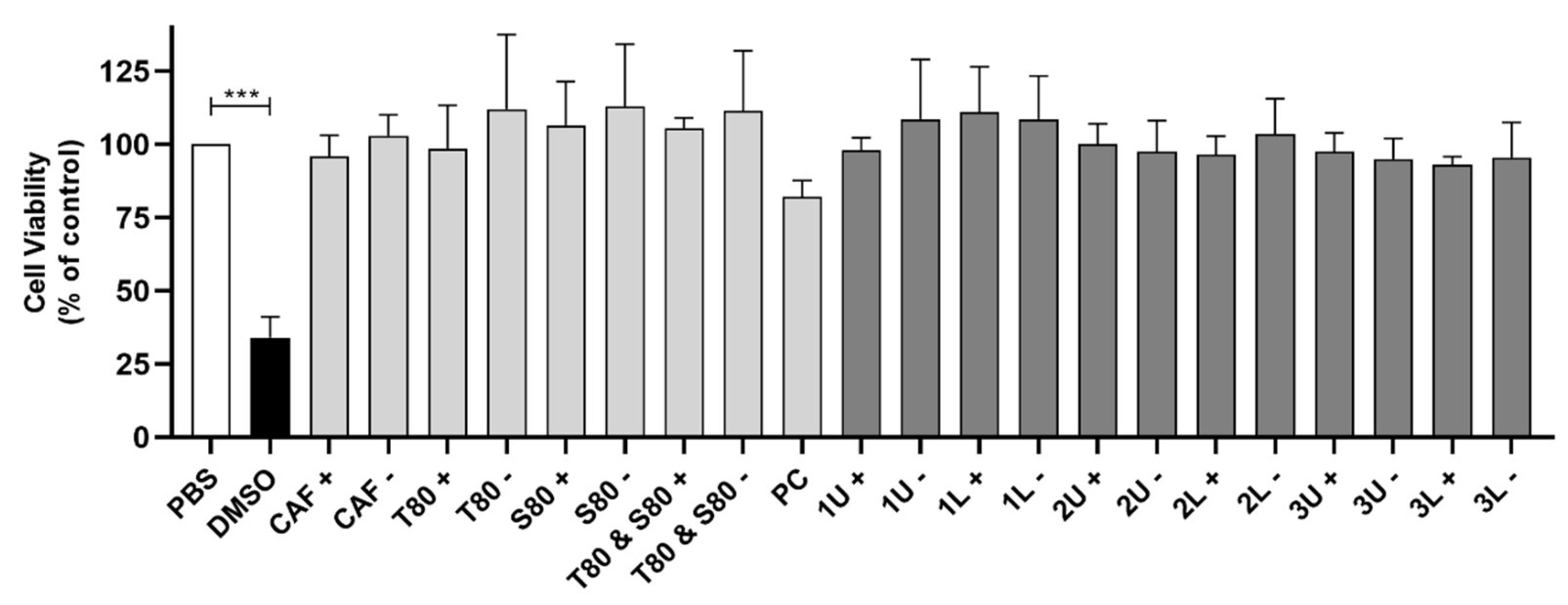

Figure 3 - Cell viability of HaCat cells after treatment for $24 \mathrm{~h}$ with the transfersomal formulations in the absence (U) and presence (L) of caffeine or with their individual constituents - caffeine (CAF), Tween ${ }^{\circledR} 80$ (T80), $\operatorname{Span}^{\circledR} 80$ (S80) or lecithin (PC).

Figura 3 - Viabilidade celular de células HaCat após tratamento por 24 h com formulações transferossomais na ausência (U) e na presença (L) de cafeína ou com os seus constituintes individuais - cafeína (CAF), Tween ${ }^{\circledR} 80$ (T80), $\operatorname{Span}^{\circledR} 80$ (S80) ou lecitina (PC).

The edge activators (EA) used in each formulation are identified as 1 (Tween ${ }^{\circledR} 80$ ), 2 (Tween ${ }^{\circledR} 80$ and Span ${ }^{\circledR}$ 80), 3 (Span ${ }^{\circledR}$ 80), and PC (no addition of EA or caffeine). DMSO 5\% (v/v) was used as positive control. The + and-symbols indicate the 1:100 and 1:200 dilution (respectively), corresponding to a caffeine concentration of 515.7 e $257.9 \mu M$ (respectively). All data were normalized considering PBStreated cells as reference and are presented as mean $\pm S D(n=2)$. The data sets with significant differences relative to control are identified with $* * * \mathrm{p}<0.001$.

$O(s)$ ativador(es) de superfície (EA) utilizados em cada formulação estão identificados por 1 (Tween $\left.{ }^{\circledR} 80\right), 2$ (Tween ${ }^{\circledR} 80$ e Span $\left.{ }^{\circledR} 80\right), 3$ $\left(\right.$ Span $\left.{ }^{\circledR} 80\right)$ e PC (sem incorporação de EA e cafeína). O DMSO 5\% (v/v) foi usado como controlo positivo. Os símbolos $+e-$ referem-se à diluição de 1:100 e 1:200 (respetivamente) em solução tampão (PBS), correspondendo a concentrações de cafeína de 515,7 e 257,9 $\mu M$ (respetivamente). Todos os dados obtidos foram normalizados considerando o controlo negativo (PBS) e estão representados como média $\pm S D(n=2)$. Os conjuntos de dados com diferenças significativas em relação ao controlo negativo estão identificados com $* * * \mathrm{p}<0,001$. 
The incorporation of Tween ${ }^{\circledR} 80, \operatorname{Span}^{\circledR} 80$, or the mixture of both in transfersomes did not affect the cytotoxicity potential of the formulations. In addition, each component of the formulations (at both concentrations under study) did not significantly reduce the viability of HaCat cells. Once again, these results are in line with previous publications regarding the incorporation of Tween ${ }^{\circledR} 80$ or $\operatorname{Span}^{\circledR} 80$ in transfersomal formulations. In particular, no cytotoxicity was reported for $\operatorname{Span}^{\circledR}$ 80-containing transfersomes after treating HaCat cells

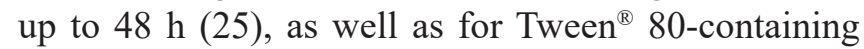
transfersomes after treating mouse melanoma cells for $24 \mathrm{~h}$ (12). To our knowledge, this is the first report on the cytocompatibility of transfersomes composed of Tween ${ }^{\circledR} 80$ and $\operatorname{Span}^{\circledR} 80$, paving the way for the development of innovative transfersomal formulations made of mixed EAs.

\section{Conclusion}

In this exploratory work, we demonstrated that transfersomes composed of single or mixed nonionic surfactants as EAs display interesting physicochemical properties for cutaneous compound delivery. Moreover, these vesicular systems were stable for one month during refrigerated storage. Although the EE and LC values of the transfersomes must be further optimized in the future, the cell viability results were also very promising, as all formulations were compatible with human keratinocytes upon $24 \mathrm{~h}$ treatment at the studied concentrations. Altogether, this study indicates that the use of mixed nonionic surfactants as EAs may be a valuable strategy to modulate the performance of transfersomes. Further studies are ongoing to determine the best ratio between nonionic surfactants in order to improve the encapsulation efficiency and the skin permeation of these vesicular systems. The later property may be further improved with the incorporation of transfersomes in a semisolid formulation, such as a gel, as it may extend the retention time on the skin.

\section{Authors Contributions Statement}

CPL, ASF, and CR, conceptualization and study design; IG, MR, and CPL, experimental implementation; IG and CPL, data analysis; IG, MR, and CPL, drafting, editing and reviewing; IG, MR, and CPL, figures and graphics; CPL, ASF, and CR, supervision and final writing. acordo com estudos anteriormente publicados que demonstraram que o tratamento de células $\mathrm{HaCat}$ com uma concentração de cafeína inferior a $512 \mu \mathrm{M}$ durante 24 h não afeta a viabilidade celular $(23,24)$.

A incorporação de Tween ${ }^{\circledR} 80$, $\operatorname{Span}^{\circledR} 80$, ou a mistura de ambos em transferossomas não alterou o potencial de citotoxicidade das formulações. Cada componente das formulações (em ambas as concentrações em estudo) também não reduziu significativamente a viabilidade das células HaCat. Mais uma vez, estes resultados estão em linha com publicações anteriores relativas à incorporação de Tween ${ }^{\circledR} 80$ ou Span ${ }^{\circledR} 80$ em formulações transferossomais. Em particular, não foi demonstrada citotoxicidade para transferossomas contendo $\mathrm{Span}^{\circledR}$ 80 após o tratamento de células HaCat até 48 h (25), bem como para transferossomas com Tween ${ }^{\circledR} 80$ após tratamento de células de melanoma de rato durante $24 \mathrm{~h}$ (12). Segundo temos conhecimento, este é o primeiro trabalho que demonstra a citocompatibilidade de transferossomas constituídos por Tween ${ }^{\circledR} 80$ e $\operatorname{Span}^{\circledR} 80$, abrindo caminho para o desenvolvimento de formulações transferossomais inovadoras constituídas por EAs mistos.

\section{Conclusões}

Neste trabalho exploratório, demonstrámos que os transferossomas constituídos por tensioativos nãoiónicos únicos ou mistos como EAs apresentam propriedades físico-químicas interessantes para veiculação cutânea. Além disso, estes sistemas vesiculares permaneceram estáveis durante 1 mês durante o armazenamento sob refrigeração. Embora os valores de EE e LC dos transferossomas devam ser ainda otimizados no futuro, os resultados de viabilidade celular foram muito promissores, uma vez que todas as formulações mostraram compatibilidade com queratinócitos humanos ao fim de $24 \mathrm{~h}$ de tratamento nas concentrações estudadas. No conjunto, este estudo indica que a utilização de tensioativos não-iónicos mistos como EAs pode ser uma estratégia valiosa para modular o desempenho dos transferossomas. Estudos adicionais estão em curso para se estudar qual será o melhor rácio entre os tensioativos não-iónicos, a fim de se otimizar a eficiência de encapsulação e a permeação cutânea destes sistemas vesiculares. Esta última propriedade pode ainda ser melhorada com a incorporação dos transferossomas numa formulação semissólida, tal como um gel, uma vez que este poderá prolongar o tempo de retenção na pele 


\section{Funding}

This study was supported by Fundação para a Ciência e a Tecnologia (FCT) through projects UIDB/04567/2020 and UIDP/04567/2020 to CBIOS and $\mathrm{PhD}$ grant 2020.07813.BD to I.G.

\section{Conflict of Interests}

The authors declare there are no financial and/or personal relationships that could present a potential conflict of interests.

\section{Declaração sobre as contribuições do autor}

CPL, ASF e CR, conceção e desenho do estudo; IG, MR e CPL, implementação experimental; IG e CPL, análise de dados; IG, MR e CPL, redação, edição e revisão; IG, MR e CPL, figuras e gráficos; CPL, ASF e $\mathrm{CR}$, supervisão e redação final.

\section{Financiamento}

Este trabalho foi financiado pela Fundação para a Ciência e a Tecnologia (FCT) através dos projetos UIDP/04567/2020 e UIDB/04567/2020 atribuídos ao CBIOS e através da bolsa de doutoramento 2020.07813. BD atribuída à I.G.

\section{Conflito de Interesses}

Os autores declaram que não há relações financeiras e/ou pessoais que possam representar um potencial conflito de interesses. 


\section{References / Referências}

1. Uche, L. E., Gooris, G. S., Beddoes, C. M., \& Bouwstra, J. A. (2019). New insight into phase behavior and permeability of skin lipid models based on sphingosine and phytosphingosine ceramides. Biochimica et Biophysica Acta - Biomembranes, 1861(7), 1317-1328. doi:10.1016/j. bbamem.2019.04.00

2. van Smeden, J., \& Bouwstra, J. A. (2016). Stratum Corneum Lipids: Their Role for the Skin Barrier Function in Healthy Subjects and Atopic Dermatitis Patients. Current Problems in Dermatologyl, 49, 8-26. doi:10.1159/000441540

3. Zhou, X., Hao, Y., Yuan, L., Pradhan, S., Shrestha, K., Pradhan, O.,Hongjie, L., Li, W. (2018). Nano-formulations for transdermal drug delivery: A review. Chinese Chemical Letters 29(12), 1713-1724. doi:10.1016/j.cclet.2018.10.037

4. Fernández-García, R., Lalatsa, A., Statts, L., Bolás-Fernández, F., Ballesteros, M. P., \& Serrano, D. R. (2020). Transferosomes as nanocarriers for drugs across the skin: Quality by design from lab to industrial scale.International Journal of Pharmaceutics, 573, 118817. doi:10.1016/j. ijpharm.2019.118817

5. Opatha, S. A. T., Titapiwatanakun, V., \& Chutoprapat, R. (2020). Transfersomes: A Promising Nanoencapsulation Technique for Transdermal Drug Delivery. Pharmaceutics, 12(9), 855. doi:10.3390/pharmaceutics12090855

6. Roberts, M. S., Mohammed, Y., Pastore, M. N., Namjoshi, S., Yousef, S., Alinaghi, A., . . Grice, J. E. (2017). Topical and cutaneous delivery using nanosystems.Journal of Controlled Release, 247, 86-105. doi:10.1016/j.jconrel.2016.12.022

7. Cevc, G., \& Blume, G. (1992). Lipid vesicles penetrate into intact skin owing to the transdermal osmotic gradients and hydration force. Biochimica et Biophysica Acta, 1104(1), 226-232. doi:10.1016/0005-2736(92)90154-e

8. Cevc, G., Gebauer, D., Stieber, J., Schätzlein, A., \& Blume, G. (1998). Ultraflexible vesicles, Transfersomes, have an extremely low pore penetration resistance and transport therapeutic amounts of insulin across the intact mammalian skin. Biochimica et Biophysica Acta, 1368(2), 201-215. doi:10.1016/s0005-2736(97)00177-6

9. Ahmed, T. A. (2015). Preparation of transfersomes encapsulating sildenafil aimed for transdermal drug delivery: Plackett-Burman design and characterization. Journal of Liposome Research, 25(1), 1-10. doi:10.3109/08982104.2014.950276

10. ElZaafarany, G. M.,Awad, G.A., Holayel, S. M., \& Mortada, N. D. (2010). Role of edge activators and surface charge in developing ultradeformable vesicles with enhanced skin delivery. International Journal of Pharmaceutics, 397(1-2), 164-172. doi:10.1016/j.ijpharm.2010.06.034

11. Jangdey, M. S., Gupta, A., Saraf, S., \& Saraf, S. (2017). Development and optimization of apigenin-loaded transfersomal system for skin cancer delivery: in vitro evaluation. Artificial Cells, Nanomedicine, and Biotechnology 45(7), 1452-1462. doi:10.1080/21691401.2016.1247850

12. Wu, P. S., Li, Y. S., Kuo, Y. C., Tsai, S. J., \& Lin, C. C. (2019). Preparation and Evaluation of Novel Transfersomes Combined with the Natural Antioxidant Resveratrol. Molecules, 24(3), 600. doi:10.3390/molecules24030600

13. Pereira-Leite, C., \& Ventura, C. (2020). Optimization of gallic acid-loaded transfersomes using a Box-Behnken factorial design. Biomedical and Biopharmaceutical Research, 17(2), 209-221. doi:10.19277/bbr.17.2.244

14. Luo, L., \& Lane, M. E. (2015). Topical and transdermal delivery of caffeine. International Journal of Pharmaceutics, 490(1-2), 155-164. doi:10.1016/j.ijpharm.2015.05.050

15. Herman, A., \& Herman, A. P. (2013). Caffeine's mechanisms of action and its cosmetic use. Skin Pharmacology and Physiology, 26(1), 8-14. doi:10.1159/000343174

16. Caparica, R., Júlio, A., Araújo, M. E. M., Baby, A. R., Fonte, P., Costa, J. G., \& Santos de Almeida, T. (2020). Anticancer Activity of Rutin and Its Combination with Ionic Liquids on Renal Cells. Biomolecules, 10(2). doi:10.3390/biom10020233

17. Júlio, A., Caparica, R., Costa Lima, S. A., Fernandes, A. S., Rosado, C., Prazeres, D., Reis, S., Santos de Almeida, T., \& Fonte, P. (2019). Ionic Liquid-Polymer Nanoparticle Hybrid Systems as New Tools to Deliver Poorly Soluble Drugs. Nanomaterials (Basel), 9(8). doi:10.3390/ nano9081148

18. Geusens, B., Van Gele, M., Braat, S., De Smedt, S. C., Stuart, M. C. A., Prow, T. W., Sanchez, W., Roberts, M.S., Sanders, N.N., Lambert, J. (2010). Flexible Nanosomes (SECosomes) Enable Efficient siRNA Delivery in Cultured Primary Skin Cells and in the Viable Epidermis of Ex Vivo Human Skin. Advanced Functional Materials, 20(23), 4077-4090. doi:10.1002/adfm.201000484

19. Danaei, M., Dehghankhold, M., Ataei, S., Hasanzadeh Davarani, F., Javanmard, R., Dokhani, A., Mozafari, M. R. (2018). Impact of Particle Size and Polydispersity Index on the Clinical Applications of Lipidic Nanocarrier Systems. Pharmaceutics, 10(2), 57. doi:10.3390/ pharmaceutics 10020057

20. Abd, E., Gomes, J., Sales, C. C., Yousef, S., Forouz, F., Telaprolu, K. C., Roberts, M. S., Grice, J. E., Lopes, P. S., Leite-Silva, V. R., \& AndréoFilho, N. (2021). Deformable liposomes as enhancer of caffeine penetration through human skin in a Franz diffusion cell test. International Journal of Cosmetic Science, 43(1), 1-10. https://doi.org/10.1111/ics.12659

21. Colletier, J. P., Chaize, B., Winterhalter, M., \& Fournier, D. (2002). Protein encapsulation in liposomes: efficiency depends on interactions between protein and phospholipid bilayer. BMC Biotechnology, 2, 9. doi:10.1186/1472-6750-2-9

22. Kapoor, B., Gupta, R., Gulati, M., Singh, S. K., Khursheed, R., \& Gupta, M. (2019). The Why, Where, Who, How, and What of the vesicular delivery systems. Advances in Colloid and Interface Science 271, 101985. doi:10.1016/j.cis.2019.07.006

23. Ojeh, N., Stojadinovic, O., Pastar, I., Sawaya, A., Yin, N., \& Tomic-Canic, M. (2016). The effects of caffeine on wound healing. International Wound Journal, 13(5), 605-613. doi:10.1111/iwj.12327

24. Xu, H., Gan, C., Gao, Z., Huang, Y., Wu, S., Zhang, D., . . Sheng, J. (2020). Caffeine Targets SIRT3 to Enhance SOD2 Activity in Mitochondria. Frontiers in Cell and Developmental Biology, 8, 822. doi:10.3389/fcell.2020.00822

25. Volkwyn, M. (2017). In Vitro Biocompatibility of Transferosomes, Ethosomes and Transethosomes. North-West University, Potchefstroom Campus, Retrieved from https://books.google.pt/books?id=Thv_ygEACAAJ 